Décadrages Décadrages

cınéma, à travers champs Cinéma, à travers champs

43 | 2020

Abdellatif Kechiche

\title{
Le regard chez Abdellatif Kechiche : un problème de méthode?
}

Coralie Lamotte

\section{(2) OpenEdition}

1 Journals

Édition électronique

URL : https://journals.openedition.org/decadrages/1575

DOI : 10.4000/decadrages. 1575

ISSN : 2297-5977

Éditeur

Association Décadrages

Édition imprimée

Date de publication : 1 juillet 2020

Pagination : 77-93

ISSN : 2235-7823

\section{Référence électronique}

Coralie Lamotte, «Le regard chez Abdellatif Kechiche : un problème de méthode ? », Décadrages [En ligne], 43 | 2020, mis en ligne le 15 octobre 2021, consulté le 03 avril 2022. URL : http://

journals.openedition.org/decadrages/1575; DOI : https://doi.org/10.4000/decadrages.1575

(B) Décadrages 
LE REGARD CHEZ ABDELLATIF KECHICHE: UN PROBLĖME DE MÉTHODE ? 

"Abdellatif Kechiche, sa méthode décryptée" (L'Express, 11 octobre 2013), "Les singulières méthodes d'Abdellatif Kechiche" (Le Parisien, 20 mars 2018), "La (méthode Kechiche ) crée des remous " (Le Monde, 4 juin 2013): les méthodes de travail d'Abdellatif Kechiche suscitent la controverse. Les articles de presse portant sur le travail du réalisateur mettent souvent en avant des techniques réputées extrêmes, voire abusives: nombre record de prises et d'heures de rush, épuisement des comédiens et des techniciens, rapports compliqués avec les producteurs... Cette critique de sa "méthode" ne constitue qu'une partie du vif débat autour du cinéma de Kechiche. Ses films eux-mêmes divisent profondément presse et spectateurs, particulièrement sur la question du regard. Pour les uns, comme pour la critique et universitaire Iris Brey s'exprimant au sujet de Mektoub, My Love: Canto Uno (France/Italie, 2017), les films de Kechiche relèvent d'une "esthétique du male gaze ${ }^{2}$, dans la mesure où ils seraient déterminés par une culture patriarcale et que les femmes y seraient regardées comme des objets, pour le plaisir d'un spectateur masculin et hétérosexuel $^{3}$. Pour les autres, comme pour le critique et écrivain François Bégaudeau, une telle "saisie féministe " ${ }^{4}$ relève avant tout d'un jugement moral et la mise en scène du regard dans ses films aurait plutôt "à voir avec la beauté du vivant ${ }^{5}$.

Dans cet article, je suggère que le regard fait partie de la «méthode Kechiche", qu'il ne relève pas seulement de problématiques de genre et ne concerne pas uniquement le rapport du spectateur au film. Le regard régit plutôt tout un réseau de relations, qui inclut les personnages, le cinéaste, les acteurs et les spectateurs: il permet de tisser des liens sur le plateau, dans la diégèse et au moment du visionnage, mais les relations qui en découlent sont souvent complexes et équivoques. Ainsi, ce commentaire des Cahiers du Cinéma à propos de la première scène d'amour de La Vie d'Adèle: Chapitres 1 et 2 (France/Belgique/Espagne, 2013) pointe les aspects problématiques du regard dans ses films: "Kechiche oublie ses personnages, il ne filme plus leurs fantasmes à elles, il ne filme plus que deux actrices soumises à sa direction. Ce qui place le spectateur dans une position insupportable: de quel droit lui infliger ça? ${ }^{6}$ Cette critique souligne le problème de hiérarchie (I'auteur parle de "soumission») que le regard du réalisateur instaure sur ses actrices, notamment lorsqu'il vient se confondre avec l'objectif de la caméra: le regard proposé par le film s'assimilerait ainsi à l'œil du cinéaste. Mais cette dimension relationnelle du regard a des effets directs sur le rapport du
1 Je remercie Felix Rietmann qui a collaboré à la mise en forme et la rédaction de certains paragraphes de cet article, ainsi qu'Achilleas Papakonstantis, Selim Krichane et Audrey Hostettler pour leurs relectures et commentaires.

2 La Grande Table (1ère partie) d'Olivia Gesbert, émission radiophonique de France Culture du 28 mars 2018, "Le désir à l'écran: il est libre, Kechiche!», 15 ${ }^{\text {ème }}$ minute, disponible en ligne: www.franceculture.fr,

3 Cf. I'article de Laura Mulvey, "Visual Pleasure and Narrative Cinema ", Screen, vol. $16, n^{\circ} 3$, automne 1975 , pp. 6-18.

4 La Grande Table, op. cit., $12^{\mathrm{ème}}$ minute.

$5 \quad / d ., 13^{\text {ème }}$ minute.

6 Jean-Philippe Tessé, "Le cœur battant", Cahiers du Cinéma, ${ }^{\circ} 693$, octobre 2013, p. 8. 
7 Pour la notion de transtextualité, j'adopte ici la définition proposée par Gérard Genette - "tout ce qui met le texte en relation, manifeste ou secrète, avec d'autres textes" - dans Palimspestes: la littérature au second degré, Paris, Éditions du Seuil, 1982, p. 7. spectateur au film: le public se retrouverait non seulement observateur consentant du désir de personnages fictifs, mais encore témoin embarrassé de la performance sexuelle de femmes bien réelles. La question des Cahiers - "de quel droit [...] infliger ça [au spectateur] ? " - ne trouve pas de réponse simple, mais elle engage à appréhender conjointement le débat sur le regard et celui sur la méthode. Pour saisir la complexité et l'ambigüité du regard, il faut repenser la «méthode Kechiche", c'est-à-dire reconsidérer les liens multiples entre discours filmique, mise en scène et spectateur.

J'aborderai cette problématique en combinant analyse des films et analyse des discours: je mènerai d'une part une étude esthétique et narrative de trois œuvres du cinéaste - L'Esquive (France, 2004), La Vie d'Adèle et Mektoub, My Love: Canto Uno - et d'autre part un examen critique d'entretiens parus dans la presse de Kechiche et de ses acteurs et techniciens. Dans un premier temps, je m'intéresserai au discours développé par ces trois films sur le sujet de la création artistique. Je montrerai que ce discours contient des dimensions transtextuelles ${ }^{7}$ et autoréflexives qui renvoient la question du regard à un tissu référentiel: celle-ci n'apparaît plus seulement comme une problématique du travail avec les acteurs, ni comme une question liée exclusivement à la place conférée au spectateur; elle doit plutôt être située au sein des liens et références entre le moment du tournage et celui du visionnage. Dans un second temps, j'examinerai les discours filmique et parafilmique sur la mise en scène, et la façon dont ils interagissent, se complètent ou se complexifient. Cela permettra de situer le regard - y compris les regards de la caméra et du spectateur - dans la structure relationnelle de la "méthode Kechiche»: le regard fonctionne comme matrice des liens entre cinéaste et acteurs, et entre films et spectateurs. La dernière partie de l'article reprendra la problématique du male gaze et proposera de l'envisager comme un aspect du discours filmique de Kechiche sur la fonction du regard dans les rapports humains: les films proposent en effet une réflexion sur le rôle clé du regard dans la création, mais surtout dans l'établissement et l'évolution des relations entre les êtres humains. Intimement lié à la création cinématographique, le regard apparaît comme un élément profondément ambigu: il permet la mise en place de relations tout en les ébranlant; il peut être productif ou destructif; il est nécessaire, mais demeure toujours problématique.

\section{LE REGARD COMME MATRICE RÉFÉRENTIELLE AU SEIN DU DISCOURS FILMIQUE}

Le thème de la création artistique parcourt l'ensemble de l'œuvre de Kechiche. II apparaît immédiatement dans les synopsis des trois films traités dans cet article. Dans L'Esquive, des collégiens d'une banlieue parisienne préparent avec leur professeure de français une représentation théâtrale de la pièce de Marivaux, Le Jeu de l'amour et du hasard (1730). Krimo, un garçon introverti, décide de s'y investir malgré son désintérêt pour l'école et le théâtre, dans le but de se 
rapprocher de Lydia, une de ses camarades. Le film thématise la question de la création via un discours sur l'adaptation et le jeu d'acteur: il retranscrit certains aspects de la pièce de Marivaux (une histoire d'amour avec des quiproquos et des mensonges, la caractérisation de milieux sociaux par le type de langage parlé) et montre les différents moments de sa mise en scène (choix des costumes et des comédiens, lecture, interprétation du texte, répétitions avec ou sans public, représentation finale). La Vie d'Adèle, adapté de la bande dessinée Le Bleu est une couleur chaude ${ }^{8}$, suit une lycéenne, Adèle, dont la vie est bouleversée lorsqu'elle rencontre Emma, étudiante aux Beaux-Arts. La première commence à travailler comme institutrice et la seconde entame une carrière d'artiste-peintre. Les deux jeunes femmes partagent une histoire d'amour pendant plusieurs années jusqu'à leur rupture, dont Adèle peine à se remettre. Leur différence de milieu social s'accompagne de relations dissemblables à l'art et la création, et cette divergence est présentée comme l'une des causes de la séparation: peindre, s'exposer et disserter sur l'art sont pour Emma des activités nécessaires à l'épanouissement de soi, tandis qu'Adèle ne se reconnaît pas dans cette conception de la création. Mektoub, My Love: Canto Uno, librement inspiré du roman La Blessure, la vraie ${ }^{9}$, raconte l'été d'Amin, un jeune homme discret, alors qu'il revient dans sa ville natale de Sète après une année passée à Paris. Venant d'interrompre ses études de médecine pour se consacrer à l'écriture scénaristique et à la photographie, il retrouve sa famille et ses amis, et fait la connaissance de vacancières qui se joignent à leur groupe. Ici encore, le film est construit autour d'une figure de créateur dont l'activité principale est d'observer les autres, et plusieurs séquences traitent explicitement du processus de création et du rôle qu'y joue le regard.

Un motif central du thème de la création, on le comprend à la lecture de ces résumés, est le recours à une multiplicité d'autres médias, comme le théâtre, la littérature, la photographie et la peinture. Le cinéma de Kechiche présente ainsi une forte dimension transtextuelle: au sein des films, divers médias et œuvres sont sollicités par l'intermédiaire de citations, références et autres procédés réflexifs comme la mise en scène de dispositifs artistiques et créatifs. Pour ne donner que quelques exemples, les personnages lisent ( Le Jeu de I'amour et du hasard dans L'Esquive, La Vie de Marianne dans La Vie d'Adèle), vont au musée et au cinéma (La Vie d'Adèle), regardent des films (Amin regarde un film de guerre soviétique dans Mektoub) et de nombreuses discussions entre deux futurs amants ont pour objet des œuvres littéraires ou artistiques.

Au niveau narratif, les procédés transtextuels encouragent la création de liens entre les personnages; au niveau esthétique, ils inscrivent l'œuvre de cinéma dans un réseau culturel et médiatique plus large. De plus, grâce à l'engagement d'un dialogue avec les œuvres convoquées, le film noue avec celles-ci des rapports thématiques. Dans La Vie d'Adèle, par exemple, la scène où Adèle voit Emma pour la première fois suit de peu une séquence où, en cours de français,
8 Julia Maroh, Le Bleu est une couleur chaude, Grenoble, Glénat, 2010.

9 Françoise Bégaudeau, La Blessure, la vraie, Paris, Verticales, 2011. 
es questions sont discutées en détail dans l'article de Cécile Sorin, "L'Esquive de Kechiche: de Shakespeare à Marivaux, analyse d'une adaptation cinématographique protéiforme", Etudes littéraires, vol. 45, n³ («Adapter le théâtre au cinéma "), Université de Laval, Canada, automne 2014, pp. 15-29.

11 Propos recueillis par JeanPhilippe Tessé, "Tomber le masque. Entretien avec Abdellatif Kechiche", Cahiers du Cinéma, n693, octobre 2013, p. 10.

12 À ce propos, voir l'article de Maud Hagelstein et Antoine Janvier, "Le problème de la vie dans le cinéma d'Abdellatif Kechiche", Cahiers du GRM, n5, 2014, disponible en ligne: http://grm.revues.org/416.

13 Le chef opérateur Sofian El Fani considère même I'«[imprégnation] par certaines images" du matériau d'origine comme "un handicap" et revendique la volonté de "s'en détacher». Propos recueillis par Cyril Béghin, «Les secrets du gros plan. Entretien avec Sofian EI Fani, chef opérateur", Cahiers du Cinéma, $n^{\circ} 693$, octobre 2013, p. 19.

14 Propos recueillis par Jacques Mandelbaum, "Abdellatif Kechiche: ‘ un artiste qui crée n'est ni homme ni femme'", Le Monde, 20 mars 2018, disponible en ligne: www.lemonde.fr. les élèves étudient le moment de la rencontre amoureuse dans La Vie de Marianne (notons aussi la similarité des titres) : I'œuvre citée vient annoncer et commenter l'évènement diégétique. De façon plus centrale encore, I'histoire et les thèmes de la pièce Le Jeu de l'amour et $d u$ hasard, commentée et mise en scène dans L'Esquive, reflètent ce qui se joue pour les personnages du film - l'impossibilité d'échapper à sa condition sociale, mais aussi le décalage entre plusieurs niveaux de langage ${ }^{10}$.

La mise en scène de l'acte d'adaptation dans L'Esquive résonne avec le discours parafilmique sur la fabrication de La Vie d'Adèle en tant que processus de création artistique. Kechiche fait ainsi appel à un véritable jeu d'enchaînements et d'imbrications de procédés transtextuels quand il explique sa méthode:

Après L'Esquive, j'avais un scénario qui développait le personnage de la professeure de français. [...] Mais je trouvais ce scénario médiocre. Puis j'ai lu la bande dessinée [Le bleu est une couleur chaude de Julia Maroh, ndlr], que j'ai trouvée passionnante. J'ai commencé à l'adapter en scénario et puis, petit à petit, j'y introduisais des éléments de ce précédent scénario, des scènes à l'école notamment, de tout cela est né un nouveau scénario où I'histoire de la BD a pris beaucoup d'importance, mais où le personnage n'a pas le même caractère. Et puis il y a des choses qui se sont mêlées, des thèmes comme celui de la prédestination de la rencontre. ${ }^{11}$

La conception de l'œuvre est abordée comme une succession de réappropriations: la bande dessinée est venue se mêler à un scénario trouvant lui-même sa source dans un autre film, ce dernier étant à son tour basé sur une pièce de théâtre. Si certains éléments dramatiques et thématiques de l'œuvre de Maroh ont été repris, de nombreuses caractéristiques narratives et esthétiques ont été laissées de côté: la structure en flashback, le caractère et les traits physiques des personnages, l'issue de I'histoire, la subjectivité de la protagoniste ${ }^{12}$, le choix du noir et blanc à l'exception de la couleur bleue pour le récit enchâssé, et la composition des cases $^{13}$. Kechiche inscrit ainsi la genèse de ses œuvres dans un tissu référentiel qui inclut des liens intraet extra-filmiques, et construit l'image d'une "méthode» de création.

C'est aussi par le biais de ce tissu référentiel qu'il répond à ses détracteurs sur sa manière de filmer les femmes:

Une femme qui danse m'émeut. Je trouve ça magnifique, vibrant, érotique, cela vient du corps et ça dépasse le corps en même temps, c'est orgasmique. Je pense d'ailleurs qu'un artiste qui crée n'est ni homme ni femme, il devient asexué. II faudrait faire, sinon, le procès aux artistes de la Renaissance, à Courbet et son Origine du monde, pourquoi pas aux sculpteurs préhistoriques des Vénus callipyges ? $^{14}$ 
La question du regard sexualisé trouve ici sa justification dans une généalogie des représentations visuelles, de la sculpture de la Préhistoire au cinéma en passant par la peinture de la Renaissance. La relation de l'artiste au sujet ne serait ainsi plus gouvernée par des questions de genre ${ }^{15}$, mais deviendrait, selon Kechiche, "asexuelle " puisqu'elle est déléguée à l'acte de création. D'après le cinéaste, le regard de l'artiste sur le sujet se situe sur un niveau qui dépasse la démarche artistique individuelle, pour devenir un élément central de la création en soi.

\section{LE REGARD COMME MATRICE DE RELATIONS DANS LA MISE EN SCÈNE}

Dans l'œuvre de Kechiche, le processus créatif fait l'objet d'une mise en scène au sein de laquelle la durée du regard occupe une fonction clé. Dans Mektoub, le personnage d'Amin est à plusieurs reprises montré en train de créer: il prend et développe des photographies, écrit un scénario. Il est seul, concentré et silencieux ${ }^{(\mathrm{Fig} .1)}$. Ces moments de "pause" sont accentués par contraste avec les autres séquences, construites autour de longs dialogues ou particulièrement chargées en termes de mouvement et de densité picturale et sonore.

Quant au discours parafilmique, la description d'une journée sur le plateau par Kechiche fait largement écho à l'image d'Amin, reprenant la tension entre mouvement et pauses: le cinéaste explique que les matinées sont généralement consacrées à un travail préparatoire, de recherche et de répétition, comme un "échauffement $"{ }^{16}$. Vient ensuite un moment solitaire, de réflexion, où "les choses commencent à se dessiner dans [s]on esprit pendant la pause déjeuner, ces deux heures durant lesquelles [il] reste seul ${ }^{17}$. Puis, le tournage se caractérise par une volonté d'enchaîner les prises, quitte à ne pas couper l'enregistrement de l'image et à ne pas utiliser de claps.

Cette question de tension entre mouvement et pauses ou, plus généralement, on le verra plus tard, de temps, est importante pour comprendre les controverses autour du jeu d'acteur chez Kechiche. C'est notamment avec L'Esquive que les discours - parafilmique et des personnages dans la diégèse - débouchent sur des stratégies de performance. Celles-ci sont discutées dans le film lorsque les collégiens préparent leur pièce de théâtre: tandis que Lydia revendique l'importance du costume ("Moi j'suis plus dans mon personnage quand j'ai ma robe») son amie Frida dénigre la valeur de cet aspect pour le jeu («On n'en a rien à branler des costumes. Pour moi, ce qu'il faut, c'est vivre les sentiments du personnage, que ça vienne de l'intérieur", [13 ème minute]). Quant à l'enseignante, exaspérée par l'introversion de Krimo, elle lui donne aussi des conseils pour améliorer son interprétation: "Amuse-toi! Tu es heureux de faire ça? II doit y avoir du plaisir à sortir de soi. [...] Donne-toi!» [71 ème minute] ${ }^{\left(\text {Fig. }^{2}\right)}$. Si I'on considère que Kechiche a affirmé, dans un entretien, son souhait de «montrer le professeur comme une métaphore du metteur en scène ${ }^{18}$ et qu'il déclare par ailleurs se sentir lui-même "plus à l'aise
15 Cela dit, cette généalogie évoquée par Kechiche est en fait elle-même "genrée" et constitue une tradition patriarcale de la représentation (des corps, notamment).

16 Propos recueillis par Philippe Rouyer, «Entretien avec Abdellatif Kechiche. Créer un état d'étourdissement ", Positif, n 632, octobre 2013, p. 20.

17 Ibid.

18 Propos recueillis par JeanMarc Lalanne, "'L'Esquive»: entretien avec Abdellatif Kechiche", Les Inrocks, 7 janvier 2004, disponible en ligne: www.lesinrocks.com. 

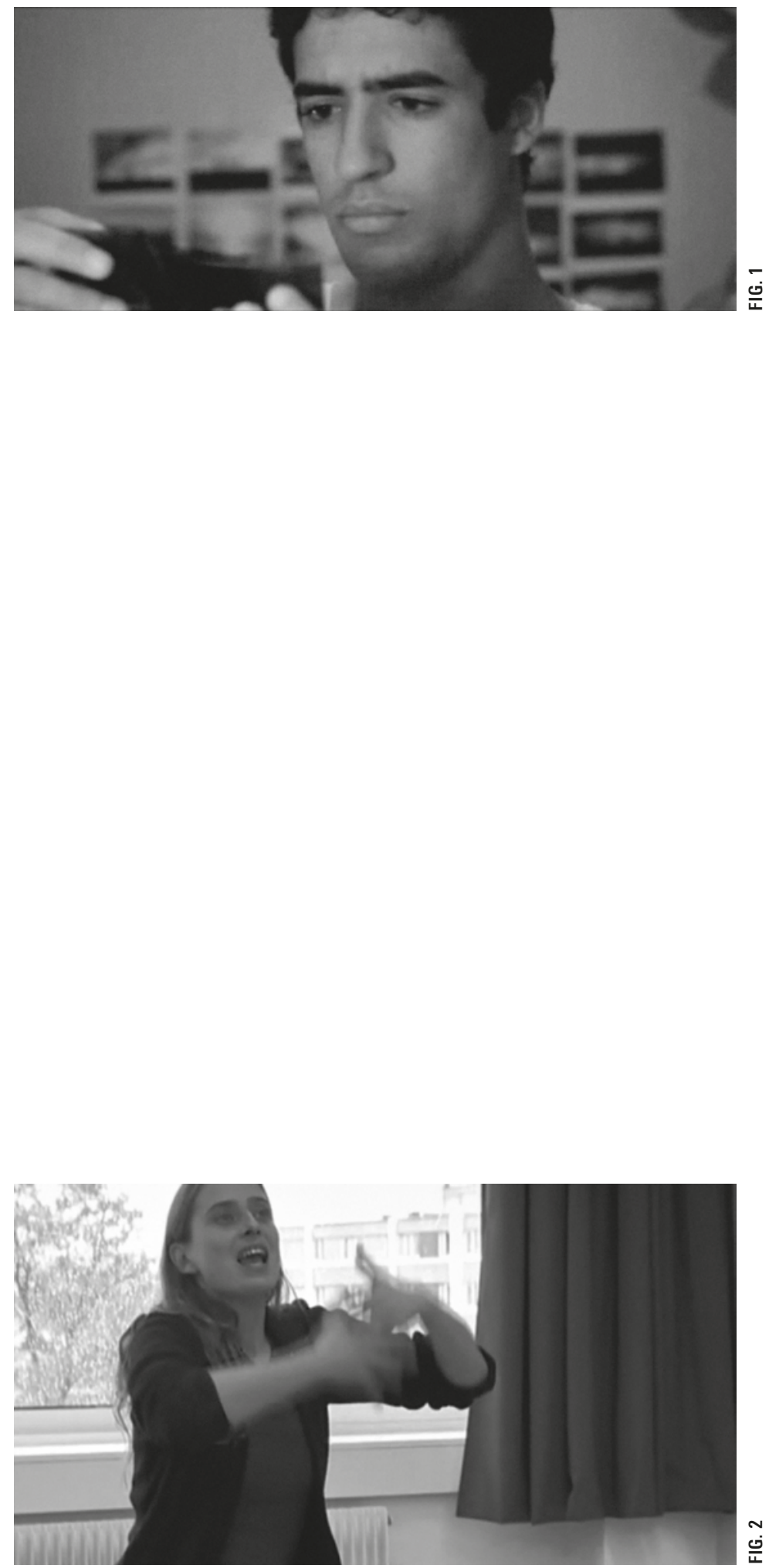
avec les acteurs qui se jettent dans le vide ${ }^{19}$, les propos de l'enseignante acquièrent un sens ambigu, résonnant avec les nombreux débats autour de la façon dont le cinéaste travaille avec ses comédiens. D'un côté, I'incitation à "se donner" suit un encouragement à s'amuser et à prendre du plaisir, et possède alors plutôt une dimension agréable et ludique. D'un autre côté, cette injonction renvoie à une prise de risques (qui rappelle l'expression "se jeter dans le vide" utilisée par Kechiche), un investissement physique et émotionnel total.

L'aspect de cet investissement qui a peut-être attiré le plus d'attention médiatique concerne la relation entre acteur et personnage. L'actrice Adèle Exarchopoulos en donne sa vision lorsqu'elle confie: "on n'a pas de maquillage, pas de coiffure: tu arrives, tu joues avec tes tripes ou c'est mort. II y a très peu de fabrication ${ }^{20}$. Hafsia Herzi, qui a tourné deux fois avec Kechiche, ajoute: «Abdellatif s'inspire toujours de nos personnalités. Bien sûr, on compose un personnage, mais il y a beaucoup de nous aussi ${ }^{21}$. Dans les films tout comme lors d'entretiens avec les actrices, la méthode de travail de Kechiche est ainsi présentée comme reposant sur l'exigence d'un type de performance qui travaille en profondeur le lien entre l'acteur et son personnage. Le scénario tient compte des interprètes: leurs prénoms sont par exemple régulièrement utilisés pour les personnages qu'ils jouent (Rachid dans L'Esquive, Adèle dans La Vie d'Adèle, Ophélie dans Mektoub), tout comme des détails de leur vie ou des aspects de leur personnalité. Selon les comédiens, ils seraient encouragés à "devenir»" ou à "se perdre avec ${ }^{23}$ leur personnage, quitte à devoir traverser des expériences physiquement et émotionnellement éprouvantes.

Une autre dimension de ces expériences concerne la relation de la performance au temps et à l'observation. Suite au scandale causé par les déclarations des actrices de La Vie d'Adèle ${ }^{24}$ sur les conditions difficiles de tournage du film, en particulier des scènes de sexe, Léa Seydoux a insisté: "Non, je ne me sentais pas libre. Quand on sait qu'on va faire cent prises, on se sent contraint, étouffé ${ }^{25}$. Hafsia Herzi est venue confirmer ce sentiment d'un étouffement par la répétition et la durée des prises en revenant sur le tournage de la scène finale de danse du ventre dans La Graine et le mulet (France, 2007): « Je ne sais même pas comment j'ai tenu, parce qu'on faisait des prises qui duraient 50 minutes. On tournait jusqu'à cinq prises par soir. $»^{26}$ Abdellatif Kechiche et Sofian El-Fani, chef opérateur avec qui il a collaboré à plusieurs reprises, confirment cet usage immodéré du temps. El-Fani explique ainsi le choix de tourner L'Esquive en numérique:

Le tournage est une sorte de recherche, jusqu'à trouver quelque chose qui s'impose. À l'époque de L'Esquive, Abdel a choisi le numérique parce que le magasin de dix minutes était trop court: dix minutes, c'est à peine la possibilité de faire venir quelque chose. Avec des durées plus longues, où l'on n'annonce pas «moteur!», où l'on ne coupe pas, le comédien a le temps d'oublier l'environnement technique. ${ }^{27}$
19 «La Master class d'Abdellatif Kechiche. Entretien avec

Pascal Mérigeau ",

3 novembre 2010, vidéo disponible en ligne: www.dailymotion.com.

20 Propos recueillis par Stéphane Delorme, "Epuisée. Entretien avec Adèle Exarchopoulos", Cahiers du Cinéma, ${ }^{\circ}$ 693, octobre 2013, p. 20.

21 Propos recueillis par Sami Gnaba, "Hafsia Herzi :

‘Abdellatif Kechiche est quelqu'un qui travaille beaucoup au ressenti. S'il ne sent pas les choses, il peut ne pas tourner '", Séquences: la revue de cinéma, $\mathrm{n}^{\circ} 314$, juin 2018, p. 8.

22 Catherine Balle, «ıMektoub my love : les singulières méthodes d'Abdellatif Kechiche", Le Parisien, 20 mars 2018, disponible en ligne: www.leparisien.fr.

23 Propos recueillis par Stéphane Delorme, "Epuisée. Entretien avec Adèle Exarchopoulos ", op. cit., p. 22.

24 Iris Mazzacurati et Sarah Lecœuvre, "La Vie d'Adèle. La polémique en 7 dates", L'Express, 25 septembre 2013, disponible en ligne: www.lexpress.fr.

25 Propos recueillis par Stéphane Delorme, "Le garçon aux cheveux bleus. Entretien avec Léa Seydoux", Cahiers du Cinéma, ${ }^{\circ} 693$, octobre 2013 , p. 24.

26 Propos recueillis par Sami Gnaba, "Hafsia Herzi : (Abdellatif Kechiche est quelqu'un qui travaille beaucoup au ressenti ı, op. cit., p. 8.

27 Propos recueillis par Cyril Béghin, "Les secrets du gros plan ", op. cit., p. 19. 
ropos recueillis par Philippe Rouyer, "Créer un état d'étourdissement", op. cit., p. 20.

29 Ibid.

30 ld., p. 21.

31 Ibid.

32 Propos recueillis par Stéphane Delorme, "Epuisée. Entretien avec Adèle Exarchopoulos", op. cit., p. 22.

33 François Bégaudeau remarque d'ailleurs: «Dans l'interminable (= ce qu'on ne saurait terminer) séquence de boîte de nuit où le film grille avec éclat ce qui lui reste de batterie, tous les cinéastes du monde ne retiendraient qu'un moment de pole dance au montage, quand même ils en auraient plusieurs en stock. Kechiche en garde quatre. Ou cinq, on ne compte plus.", François Bégaudeau, "La lumière et c'est tout", Transfuge, $\mathrm{n}^{\circ} 118$, avril 2018, disponible en ligne: www.transfuge.fr. On pourrait alors compare cette expérience à ce qu'Alexander Sesonske appelle "notre conception au sens commun du temps" et définit ainsi : "Le temps est continu [...]. ‘Continu ` signifie que tout point dans le temps est connecté avec tous les autres via des points (moments) intermédiaires et que l'on ne peut passer d'un moment à l'autre qu'en traversant les moments intermédiaires." Alexander Sesonske, "Time and Tense in Cinema", The Journal of Aesthetics and Art Criticism, vol. 3, $\mathrm{n}^{\circ} 4$, été 1980, p. 419 [ma traduction].
Cependant, Kechiche fait part d'une vision sensiblement différente du vécu des comédiennes: il présente cet usage du temps comme libérateur, permettant d'«allège[r] les tensions et les pressions sur les acteurs ${ }^{28}$. II revendique ainsi l'intérêt de travailler dans la durée, qui aurait pour bénéfice de "crée[r] une sorte d'étourdissement et gomme[r] la notion de jeu ${ }^{29}$.

La durée ne se limite pas à celle d'une performance car elle a lieu devant la caméra, ce qui implique un temps de regard et d'exposition au regard. C'est la durée de cette exposition à la caméra (et par extension, au regard du cinéaste et à celui du spectateur) qui est censée amener à un "étouffement" des acteurs. La présence du regard est considérée comme indispensable: certaines scènes de La Vie d'Adèle auraient ainsi eu pour seul but d'«offrir aux actrices du vécu de leur personnages ${ }^{30}$ et n'avaient jamais été conçues pour être conservées au montage. Kechiche a d'ailleurs souligné qu'il «[s]e gardai[t] bien de le dire aux actrices ${ }^{31}$. Les acteurs doivent épuiser leurs ressources, et ce, devant la caméra, jusqu'à l'oubli des conditions de possibilité de cette performance. Adèle Exarchopoulos déclare ainsi : "Il y avait tellement de rushes que je ne savais pas quel film j'allais me prendre dans la figure. [...] On avait cette liberté qui pouvait aussi être dangereuse. On s'habituait aux caméras, on les oubliait complètement ${ }^{32}$.

Ce regard dans la durée configure non seulement le rapport entre le cinéaste (ou plus largement l'équipe de tournage) et les acteurs - via la performance - mais aussi celui entre les spectateurs et le film, au moment du visionnage. Remarquables par leur durée (entre deux et trois heures pour la plupart), les films présentent tous des séquences très longues et répétitives: la danse du ventre de La Graine et le mulet, les scènes de sexe de La Vie d'Adèle, les discussions-rabâchages de L'Esquive (par exemple, lorsque Fathi prend le téléphone de Frida, où I'un répète inlassablement "arrête de crier» et l'autre "rends-moi mon portable» [76 ${ }^{\text {ème }}$ minute]), et les séquences de vingt minutes dans Mektoub, comme celles à la plage et en boîte de nuit, qui semblent affranchies de toute convention narrative. Ces passages fournissent une expérience inhabituelle du temps au cinéma ${ }^{33}$. Les séquences s'étirent sans donner aucun indice sur leur durée ou leur issue, mais le spectateur doit les éprouver en visionnant le film ${ }^{34}$ - tout comme les acteurs doivent éprouver les prises de vue lors du tournage. Le regard «interminable» qui intervient au moment du tournage se répercute sur les spectateurs pendant la projection: ceux-ci sont plongés dans l'expérience d'un temps présent partagé, soumis aux rythmes lents et répétitifs des personnages, parfois jusqu'à un point presque insoutenable, comme l'exprimait la critique des Cahiers citée plus haut à propos de la première scène de sexe de La Vie d'Adèle. Cet aspect est mis en scène dans L'Esquive, lors d'une séquence particulièrement difficile qui montre une répétition de la pièce en classe: Krimo et Lydia, dans leurs costumes d'Arlequin et de Lisette, jouent une scène face à l'enseignante et aux autres élèves. Alors que Lydia maîtrise son texte et l'interprète de façon "juste " et expressive, Krimo trébuche sur les mots, marmonne d'une voix monocorde, ne sait pas 
où regarder et semble encombré par son propre corps. L'enseignante prodigue, sans succès, des conseils à Krimo, et l'apathie du garçon finit par la mettre hors d'elle: elle crie et s'agite, jusqu'à ce qu'il quitte la salle. Les élèves, eux, observent la scène dans un silence accablant. Le poids du regard, soutenu par la durée de la scène et accompagné du discours de la professeure, de plus en plus frénétique et irritée, devient insupportable pour tout le monde, jusqu'à parvenir à un point de rupture: Krimo préfère se dérober et partir, les élèves, y compris Lydia, continuant à observer la scène avec embarras; quant à l'enseignante, elle baisse les yeux après le départ de Krimo. Le spectateur du film hérite du poids de ce regard, vivant lui aussi cette scène comme un moment difficile. L'intransigeance dans le traitement du temps vise ainsi autant à l'épuisement des acteurs et des scènes qu'à celui des spectateurs. Selon Kechiche, la durée du regard permettrait au public d'atteindre un état "participatif ${ }^{35}$, propice au visionnage. La durée des scènes, parfois difficilement supportable comme dans cette séquence de $L^{\prime} E$ squive qui met en scène un public face à un spectacle (ici, une répétition), renvoie alors le spectateur à lui-même et à son propre regard.

Cette relation réflexive entretenue avec le spectateur - par le biais d'un regard continu, se déployant dans la durée - est soutenue par de multiples métaphores mettant en abyme le dispositif cinématographique. Par exemple, à la fin de L'Esquive, Fathi organise une rencontre entre Krimo et Lydia dans une voiture afin que celle-ci réponde à la demande du garçon de sortir avec lui. Fathi et les deux amies de Lydia s'assoient en face du véhicule, sur la plateforme d'une remorque, légèrement en surplomb. Public investi, ils observent, commentent et

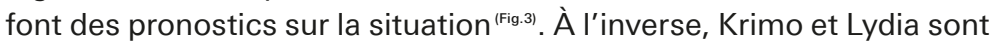
paralysés. Après un moment de silence, Krimo initie timidement la discussion, mais Lydia, pourtant à l'aise sur la scène de théâtre, explose en manifestant son angoisse et son refus de répondre sous la pression qu'on lui impose; Krimo, lui, n'ose plus parler. Sa seule tentative est interrompue par Lydia, avant que la police ne débarque et mette violemment fin à la discussion. Alors qu'ils sont à "l'écran" (le pare-brise de la voiture), les personnages sont statiques, incapables de dialoguer

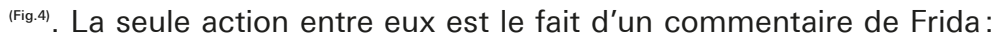
«Eh, elle lui a pris la main!» [98 ème minute], qui fait instantanément réagir les deux autres, mais aucune confirmation n'est apportée au spectateur par le contrechamp. Fathi et les amies de Lydia acquièrent, en tant qu'observateurs et grâce à leur participation active, autant d'importance que Krimo et Lydia, pourtant supposés être les protagonistes. L'attente, la distance spatiale et la durée (les trois adolescents patientent, assis un peu à l'écart, guettant l'avancée de la discussion) encouragent leur participation à l'action, provoquent l'événement et font écho à l'expérience du spectateur.

Ainsi, la durée et la structure relationnelle du regard, tant au niveau du tournage que du visionnage du film, configurent les liens acteurpersonnage et spectateur-film, en concrétisant une certaine image bien que potentiellement provocatrice et éthiquement problématique 

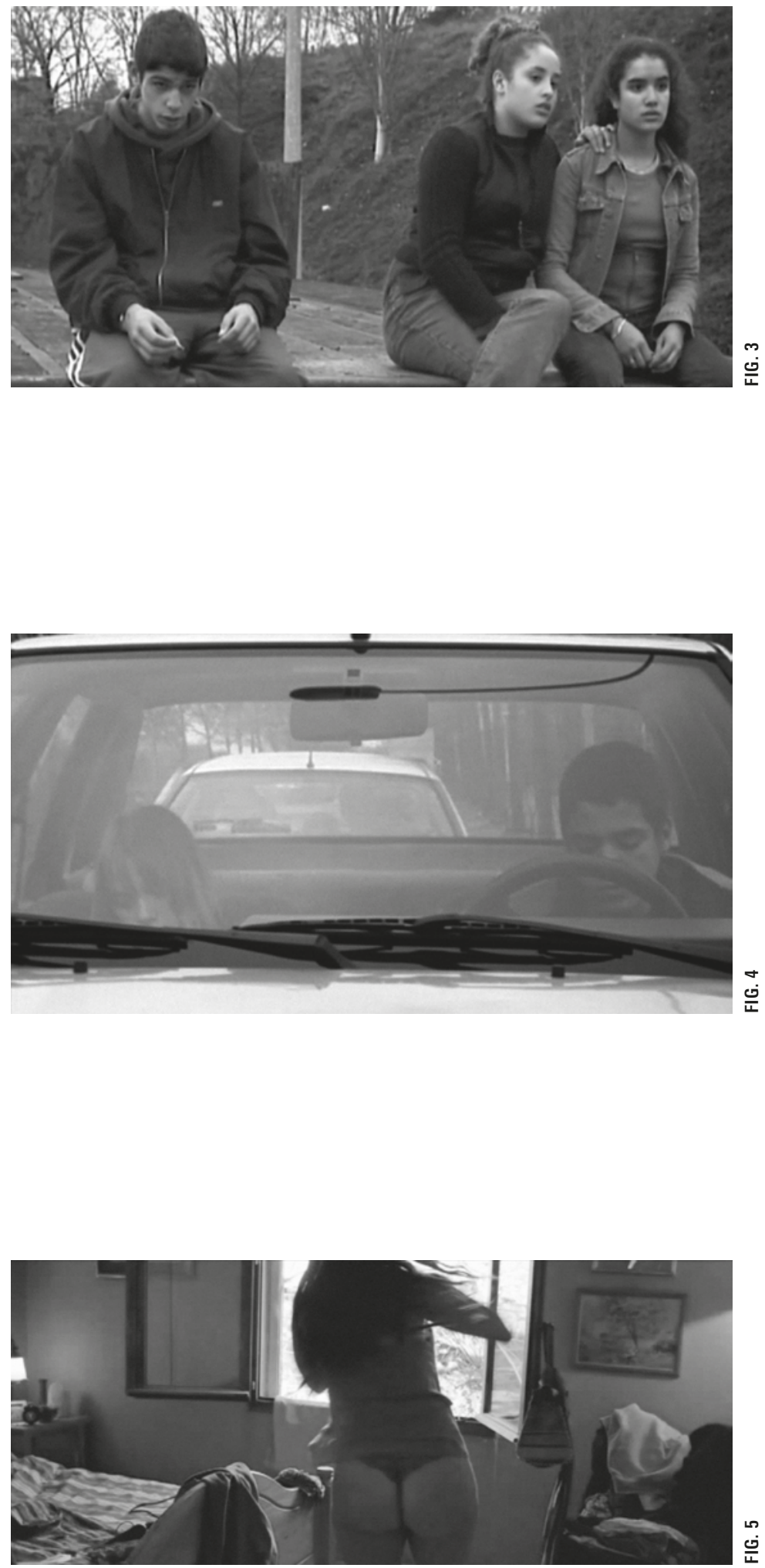
- de la performance des acteurs et de la participation du public. Le regard ne permet pas seulement d'établir et de maintenir des relations, mais il joue aussi un rôle d'agent perturbateur au sein des rapports qu'il instaure: sa durée et son insistance le rendent parfois difficilement supportable, tant pour les personnes regardées que pour les spectateurs.

\section{LE REGARD VOYEURISTE COMME MÉTHODE?}

L'effet perturbateur du regard constitue un thème récurrent dans la réception critique des films de Kechiche, tant sur le plan de ses méthodes de travail avec les actrices que sur celui de leur fonction esthétique et narrative. Une partie de la critique s'est concentrée sur les enjeux de pouvoir du regard genré, le "male gaze » déjà mentionné ${ }^{36}$. Kechiche, quant à lui, semble d'abord pleinement assumer, et même valoriser, le plaisir de celui qui porte son regard sur un sujet filmé, associant regard et participation:

Si je braque l'œil de la caméra sur un corps, c'est que j'ai envie de voir. II y a voyeurisme, pas au sens péjoratif, mais regarder le corps de ces femmes est une expérience par laquelle on est saisi. [...] On est voyeur à partir du moment où I'on refuse de s'impliquer. [...] En revanche, si je veux voir et participer de tout mon être, de tout mon corps, au risque d'en perdre la raison dans une sorte d'étourdissement, ce sera différent. ${ }^{37}$

Le regard voyeuriste, s'il est accompagné d'une participation totale, serait ainsi justifié; il devrait même être avide et sans réserve afin que I'observateur vive une authentique expérience physique et émotionnelle. Pourtant, la citation souligne ici encore la répartition genrée du regard: le réalisateur, qui est un homme et affirme sa position d'observateur ("je», qui devient ensuite "on ", l'expérience de l'homme étant ainsi généralisée), parle du "corps des femmes». Ses films abondent de gros plans sur de jeunes femmes, leurs seins et leurs fesses, sans que ceux-ci soient attribuables au point de vue d'un personnage. Iris Brey remarque ainsi, à propos d'une scène de Mektoub dans laquelle

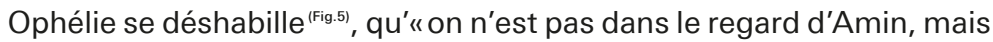
on est dans le regard de la caméra [...] qui regarde un beau cul comme un objet de désir ${ }^{38}$. Krauthaker et Connolly pointent également, au sujet de La Vie d'Adèle, cette problématique de I'“objectification » du corps d'Adèle, sur lequel la caméra "demeure obsessionnellement fixée ", ce qui rend "certaines scènes embarrassantes à regarder " ${ }^{39}$.

Mais les films semblent en fait jouer sur plusieurs registres, en mettant eux-mêmes en scène un discours très ambivalent sur le regard. Ils intègrent par exemple une réflexion sur la fonction du regard dans le processus de création qui accentue la dimension du temps et de la durée. Mektoub propose une image du créateur semblable à celle revendiquée par Kechiche dans ses entretiens: un artiste attentif, réceptif aux évènements, guidé avant tout par son regard et son intuition. Dans le film, Amin se rend à la ferme d'Ophélie avec l'espoir
Cf. notes 2 et 3 et lire par exemple l'entretien de Julia Maroh à propos de La Vie d'Adèle, dans Le Parisien (propos recueillis par N. VE.): "Julia Maroh, La Vie d'Adèle, "une vision hétéro "), 3 octobre 2013, disponible en ligne: www.leparisien.fr. Egalement la critique de Mektoub, My Love: Canto Uno par Hugues Dayez, 2 mai 2018, disponible en ligne: www.rtbf.be, ou celle d'Anaïs Bordage pour Mektoub, My Love: Intermezzo, "Vous ne savez pas ce qu'est le (male gaze)? II suffit de voir le film de Kechiche", 24 mai 2019, disponible en ligne: www.slate.fr.

37 Propos recueillis par Philippe Rouyer, "Créer un état d'étourdissement", op. cit., p. 22.

38 La Grande Table, op. cit., 14 ème minute.

39 Marion Krauthaker et Roy Connolly, "Gazing at Medusa: Adaptation as phallocentric dynamics in Blue is the Warmest Colour", Comics and Adaptation in the European Context Friday, University of Leicester, 2017, disponible en ligne: http://hdl.handle.net/ 2381/41917, p. 29 [ma traduction]. 

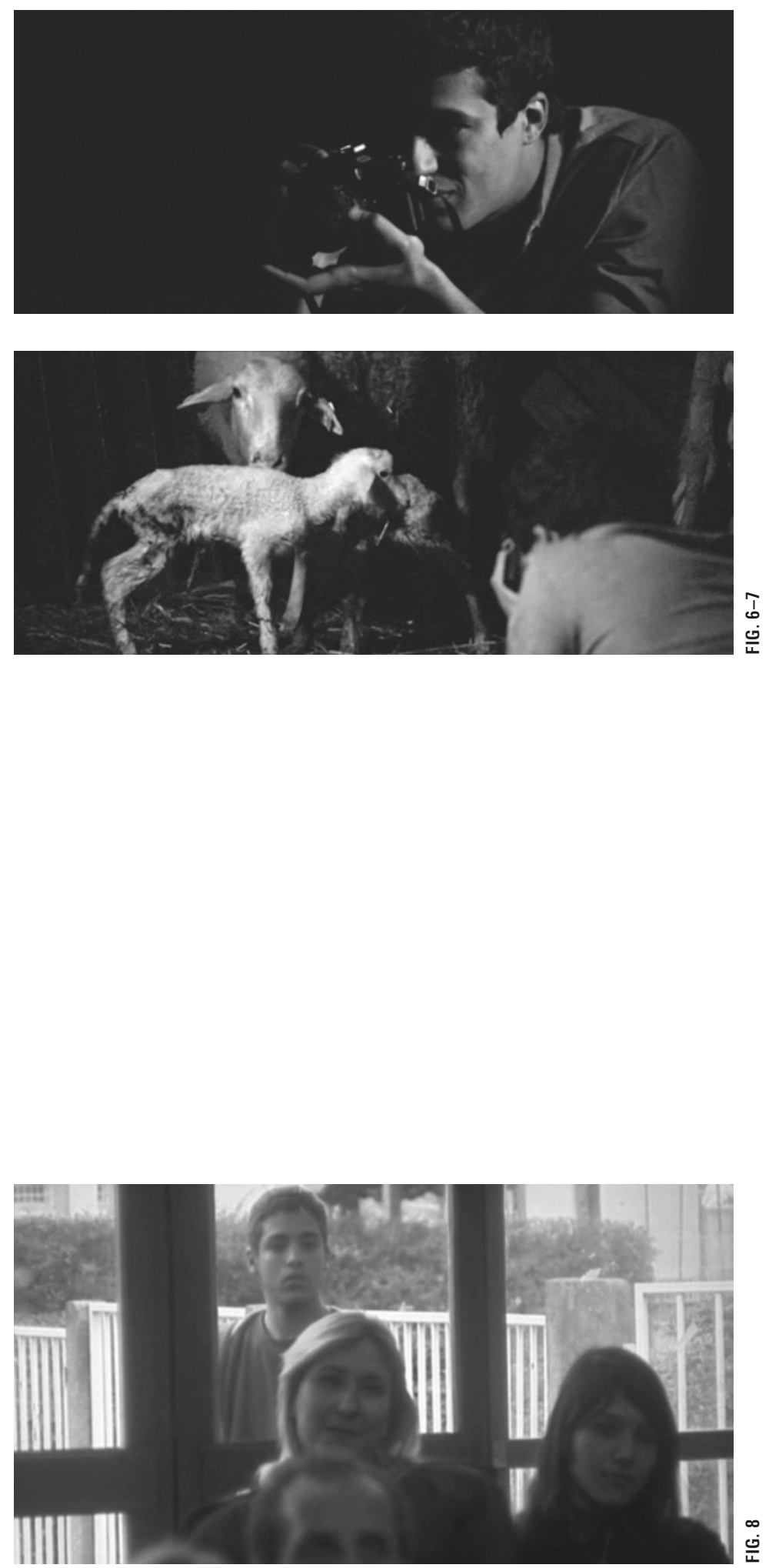
d'assister à la naissance d'agneaux et de photographier ce moment. Après avoir écouté les conseils de son amie, il déclare: "Je vais me mettre là, tranquille, les observer, et attendre" [129ème minute]. Resté seul avec les brebis, il suit ce protocole et patiente longuement. Le soleil se couche et, sous l'objectif et le regard fascinés d'Amin, une brebis met au monde un premier agneau, puis, surprenant à la fois le spectateur et le personnage, un second ${ }^{(\text {fig.6-7) }}$. Si l'on en croit Kechiche, qui explique: "Je laisse venir les choses à moi, et j'ai de plus en plus confiance dans le fait que le film va choisir sa direction ${ }^{40}$, cette scène serait révélatrice de sa propre façon de travailler. Elle peut quoi qu'il en soit être lue comme une réflexion sur la patience et l'investissement du regard dans la démarche artistique.

Le regard peut aussi être dysfonctionnel, détruire les rapports humains et faire barrage à la création. Dans L'Esquive, par exemple, Krimo est présenté comme un personnage qui n'arrive pas à avoir un rôle et un regard actifs, ce que cristallise sa position d'incompréhension, voire de passivité, vis-à-vis de diverses formes d'art. Désemparé face à la littérature classique à laquelle il s'attèle dans le but de séduire Lydia, il avoue son incapacité à comprendre Marivaux, expliquant que les phrases sont trop longues et le vocabulaire complexe. En outre, s'il reçoit des dessins de bateaux de la part de son père prisonnier et les affiche dans sa chambre, c'est son ami Fathi qui les prend comme une invitation au voyage, à la rêverie et à une vie meilleure. Au début du film, Lydia doit le forcer pour qu'il accepte d'assister à la répétition de théâtre, mais Frida, l'amie et partenaire de jeu de Lydia, refuse sa présence et critique son attitude de spectateur: "II y a des façons de regarder. II se moque " [19ème minute], dit-elle, alors que Krimo observe toute la scène avec impassibilité. Mal à l'aise, il finit par s'en aller sans se défendre. Lors de la représentation finale, il n'est pas présent. Spectateur distant, il s'approche seulement de la salle pour jeter un coup d'œil à travers la vitre avant de s'éloigner ${ }^{(\mathrm{Fig} .8)}$. Pourtant, c'est de sa propre initiative que Krimo s'investit dans la préparation de la pièce et demande à l'interprète d'Arlequin de lui céder son rôle. Mais les quelques répétitions auxquelles il participe sont un échec: Krimo ne parvient pas à sortir de son mutisme et de son introversion malgré les exhortations de Lydia et de sa professeure. Rapidement, il finit par abandonner. Son incapacité à assumer le regard - comme sujet ou objet de celui-ci - l'empêche de mener à bien son projet de théâtre et sa relation avec Lydia, I'isolant littéralement: I'un des derniers plans du film le montre seul dans sa chambre, muet et caché derrière le rideau lorsque Lydia l'appelle.

La réflexion sur le regard devient explicitement ambigüe lorsqu'elle touche au vécu du sujet comme objet du regard et sa relation avec celui qui regarde, c'est-à-dire aux dimensions relationnelles du regard. Dans Mektoub, lorsqu'Amin demande à Ophélie de poser nue pour lui et qu'elle refuse, il insiste: "C'est plaisant, non? T'auras des photos de toi, à ton avantage, ton corps sera mis en valeur... Ça va te plaire." Ce à quoi Ophélie répond: "C'est plaisant pour toi, quoi. Non, mais je ne vois pas I'intérêt " [117 ${ }^{\text {ème }}$ minute]. Avant de finalement Philippe Tessé, «Tomber le masque. Entretien avec Abdellatif Kechiche», op. cit., p. 10. 

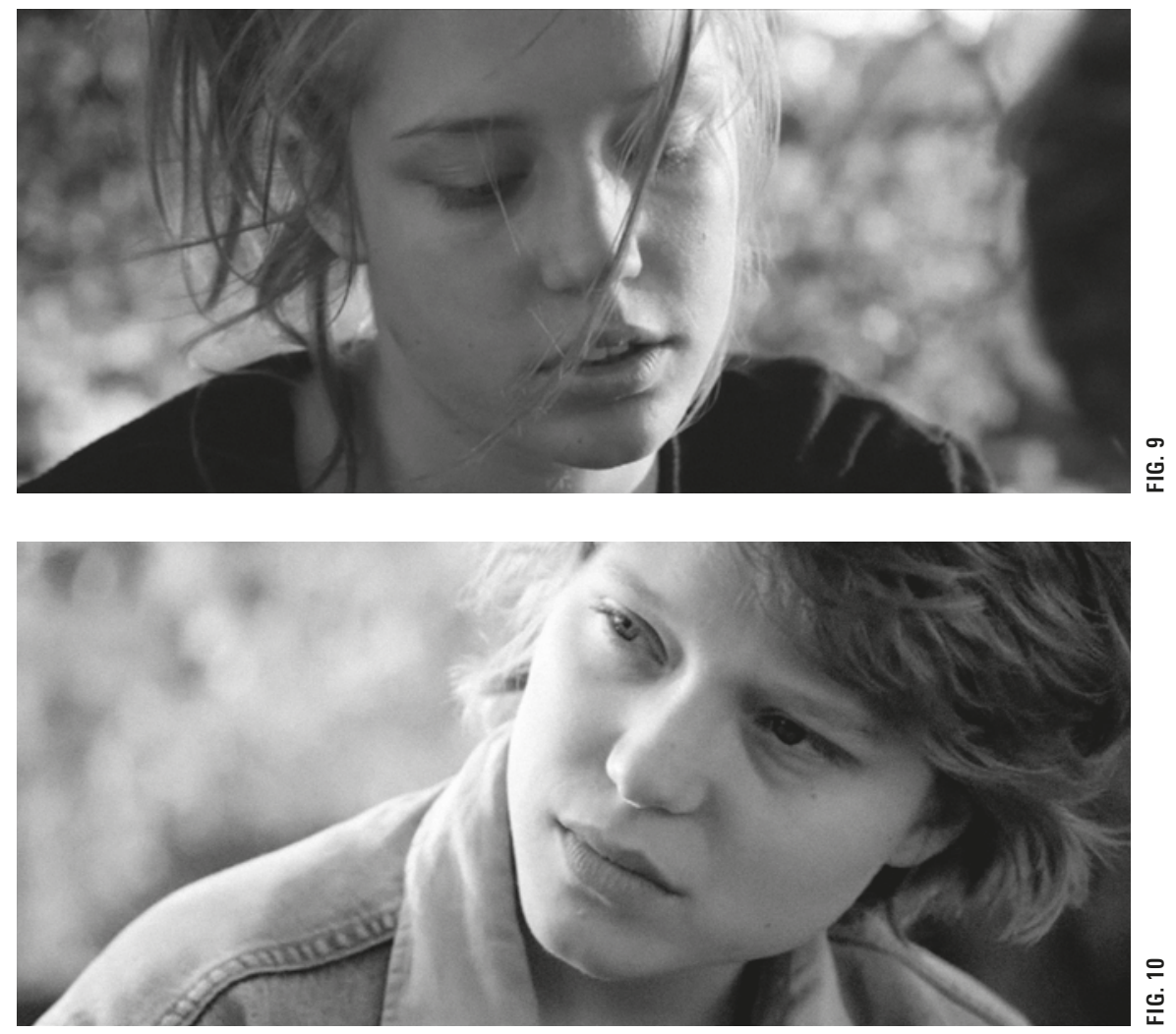
revenir sur son refus, gênée mais curieuse et flattée: "Non, non. Alors... Je fais quoi, en fait, je me mets comment?" [118 ème minute]. Pour les spectateurs, qui savent qu'Amin a observé Ophélie en pleins ébats sexuels (et l'ont par la même occasion également vue nue sous tous les angles), cette molle opposition semble presque ironique, tout en soulignant les tensions potentielles entre vécu subjectif et objectification. Plus tard dans le film, Ophélie, laissant Amin avec ses brebis, lui glisse: "Si tu fais bien tes photos, peut-être que j'accepterai de poser nue pour toi " [131 ${ }^{\text {ème }}$ minute]. Acceptant finalement de soumettre son corps au regard et à l'appareil photo d'Amin, Ophélie, en posant cette condition, fait aussi évoluer sa position: elle sera objet du regard d'Amin, mais d'abord juge de la qualité de celui-ci, spectatrice du travail du jeune homme avant d'être son modèle (à nouveau, car le film nous apprend qu'elle a déjà posé pour lui). Le regard de l'observateur masculin se retrouve donc lui-même soumis au regard de la femme regardée, mettant en place un jeu relationnel à la fois ludique et sérieux - puisque la relation entre Ophélie et Amin est toujours ambigüe dans le film, entre amitié et séduction - guidé par le regard.

En même temps, ce discours filmique peut se lire comme un commentaire sur les controverses autour des méthodes de travail de Kechiche. II s'agirait d'une mise en scène de la tension entre les dires du cinéaste, qui voit sa technique de tournage comme bénéfique et agréable, et affirme que les "actrices sont parfaitement consentantes " ${ }^{41}$, et les dires des comédiens, et surtout des comédiennes, qui affichent des propos bien plus mitigés sur leur expérience. Bien que le plaisir de l'observateur soit partiellement légitimé, entre autres en encourageant la personne regardée à apprécier elle aussi sa position, voire en la soupçonnant d'en tirer de la satisfaction, la question de la gêne et du plaisir reste ambivalente et ouverte autant dans les films que pour les spectateurs. Dans La Vie d'Adèle, lorsqu'Emma dessine le portrait d'Adèle et remarque sa nervosité, elle lui dit ainsi: "T'es gênée? [...] C'est vrai? Ça ne te fait pas plaisir? " [53 ${ }^{\text {ème }}$ minute] (Fig.9-10)

Le regard interroge. Il est présenté à la fois comme essentiel dans la démarche créative et ambigu dans les relations entre les personnages. II structure les rapports humains tout en instaurant des hiérarchies: si le regardeur semble majoritairement avoir l'emprise sur le regardé (l'air inquisiteur d'Emma qui examine en détail son modèle versus l'embarras d'Adèle qui se traduit par son regard inconstant) et que la répartition est souvent genrée (I'homme regarde, la femme est regardée), les films et les discours montrent des ruptures et des renversements. La frontière entre plaisir et malaise, entre jeu et sérieux, entre subjectivité et objectification, n'est jamais clairement établie. Faisant intégralement partie de la "méthode Kechiche", le regard est créateur, parfois dysfonctionnel, parfois productif, mais presque toujours problématique.
41 Propos recueillis par Jacques Mandelbaum, "Abdellatif

Kechiche: ‘ un artiste qui crée n'est ni homme ni femme'",, op. cit. 